\title{
Stochastic thermodynamics for linear kinetic equations
}

\author{
C. Van den Broeck \\ Hasselt University, B-3590 Diepenbeek, Belgium \\ R. Toral \\ Instituto de Física Interdisciplinar y Sistemas Complejos (IFISC), Campus UIB, Palma de Mallorca, Spain
}

(Received 31 March 2015; published 22 July 2015)

\begin{abstract}
Stochastic thermodynamics is formulated for variables that are odd under time reversal. The invariance under spatial rotation of the collision rates due to the isotropy of the heat bath is shown to be a crucial ingredient. An alternative detailed fluctuation theorem is derived, expressed solely in terms of forward statistics. It is illustrated for a linear kinetic equation with kangaroo rates.
\end{abstract}

DOI: 10.1103/PhysRevE.92.012127

PACS number(s): 05.70.Ln, 05.40.-a

\section{INTRODUCTION}

The second law of thermodynamics is arguably one of the most general laws of nature. While originally stipulating the increase of total entropy in a closed isolated system $\Delta S_{\text {tot }} \geqslant 0$, it was reformulated by splitting the entropy change $\Delta S$ of an open system into the sum $\Delta S=\Delta_{i} S+\Delta_{e} S$ of a nonnegative entropy production term $\Delta_{i} S \geqslant 0$ plus an entropy exchange contribution $\Delta_{e} S$. In particular when in contact with a single heat bath at temperature $T$, the exchange is given by $\Delta_{e} S=$ $Q / T$, where $Q$ is the amount of heat into the system. Over the past two decades, a much deeper formulation of the second law has been achieved by focusing on small open systems. One can still define all the above-mentioned quantities, but they will now fluctuate from one measurement to another. Using lower case to distinguish the values from the nonfluctuating macroscopic counterparts, one has $\Delta s=\Delta_{i} s+\Delta_{e} s$, with $\Delta_{e} s=q / T$. The second law is replaced by a symmetry property for the probability density $P\left(\Delta_{i} s\right)$ to observe an entropy production $\Delta_{i} s$. In its simplest form, the so-called fluctuation theorem states that the probability for observing an entropy increase is exponentially larger than that for observing a corresponding decrease, $P\left(\Delta_{i} s\right)=\exp \left(\Delta_{i} s / k_{B}\right) P\left(-\Delta_{i} s\right)$, being $k_{B}$ Boltzmann's constant. The second law $\left\langle\Delta_{i} s\right\rangle \geqslant 0$ follows as a subsidiary result. The fluctuation theorem has been obtained at different levels of description, ranging from the microscopic laws [1,2], over thermostated systems [3,4] to stochastic dynamics [5-7]. "Stochastic thermodynamics" is easy to formulate in the context of a Markovian description, both at the level of a Langevin and Fokker-Planck equation or the more general Master equation [8-11], and its predictions have by now been confirmed by numerous experiments. The focus has been mostly on overdamped systems with variables that are even under time-reversal. However, for variables, such as velocities instead of positions, it was claimed that the theory becomes more involved and hence loses some of its appeal $[12,13]$. In this paper, we show that this is not the case if the transition probabilities obey, in addition to detailed balance, a symmetry property, reflecting the fact that the heat bath is not in motion with respect to the system and that it is isotropic. To demonstrate the role and importance of this condition, we develop the stochastic thermodynamics, both at the ensemble and trajectory level, for linear kinetic equations, a field for which there is a large potential interest. We derive the fluctuation theorem, including a new version expressed only in terms of probabilities computed from the forward process. As an application, we provide explicit illustrations for the special case of kinetic "kangaroo" equations $[14,15]$. The role of a general parity symmetry condition similar to the one used here has also recently been pointed out in the context of the quantum Jarzynski equality [16], in the sense that the equality does not hold if parity is violated.

\section{STOCHASTIC THERMODYNAMICS FOR KINETIC EQUATIONS}

We consider the simplest scenario of a system consisting of a single stochastic Maxwell-Lorentz particle, cf. Refs. [17,18] for a detailed analysis of a similar model, with mass $m$, velocity $v$ at position $x$ in the constant external force field $F$ (acceleration $a=F / m$ ), and in contact with a single isotropic heat reservoir at rest with temperature $T$. The stochastic dynamics of the particle is characterized by a probability density $P(x, v ; t)$, obeying the linear kinetic equation:

$$
\begin{aligned}
& {\left[\frac{\partial}{\partial t}+v \frac{\partial}{\partial x}+a \frac{\partial}{\partial v}\right] P(x, v ; t)} \\
& \quad=\int d v^{\prime}\left[k\left(v^{\prime} \rightarrow v\right) P\left(x, v^{\prime} ; t\right)-k\left(v \rightarrow v^{\prime}\right) P(x, v ; t)\right] .
\end{aligned}
$$

Here $k\left(v^{\prime} \rightarrow v\right)$ is the transition probability per unit time (rate) for a change of velocity from $v^{\prime}$ to $v$. A stochastic kinetic description has also been studied in detail for the so-called adiabatic piston, for recent references see Refs. [19,20].

Formulation of the first law at the trajectory level is straightforward. The energy $e(t)$ of a particle in the constant external force field $F$ is

$$
e(t)=-F x(t)+\frac{1}{2} m v^{2}(t),
$$

where $x(t)$ and $v(t)$ are the position and velocity of the particle at time $t$ in the given realization. The "ensemble" version of the first law is obtained by averaging with respect to the probability density $P(x, v ; t)$ :

$$
E(t)=\langle e(t)\rangle=-F\langle x(t)\rangle+\frac{1}{2} m\left\langle v^{2}(t)\right\rangle .
$$

In-between collisions, potential energy is converted into kinetic energy following Newton's law $m \dot{v}(t)=F$, hence this nondissipative process produces no net energy $\dot{e}(t)=0$, and 
neither work nor heat are exchanged. The punctual collisions with the heat bath, however, lead to an instantaneous exchange of energy under the form of heat:

$$
\dot{e}(t)=\dot{q}(t),
$$

with $\dot{q}(t)$ a sum of $\delta$ functions at the instants of the collision and with amplitude $\frac{1}{2} m\left(v^{2}-v^{\prime 2}\right)$ for a collision changing the velocity from $v^{\prime}$ to $v$. At the ensemble level, the resulting heat flux $\dot{Q}(t)$ is obtained by averaging over the frequency of such collisions:

$$
\dot{E}(t)=\dot{Q}(t)=\iint d v d v^{\prime} k\left(v^{\prime} \rightarrow v\right) P\left(v^{\prime}, t\right) \frac{1}{2} m\left(v^{2}-v^{\prime 2}\right) .
$$

We next turn to the second law and formulate it first at the ensemble level. In principle, the "ensemble" entropy associated to the distribution $P(x, v ; t)$ is given by $S(t)=$ $-k_{B} \int d x d v P(x, v ; t) \ln P(x, v ; t)$. However, when considering the time derivative of this quantity, we note that the motion is purely Hamiltonian in-between collisions. Following Liouville's theorem, this part of the dynamics leaves the entropy invariant [21]. Hence, we need only to focus on the change of the entropy induced by the dissipative collisions, affecting solely the velocity variables. From

$$
S(t)=-k_{B} \int d v P(v, t) \ln P(v, t),
$$

we find in combination with the evolution equation for $P(v, t)$, obtained from Eq. (1), and following some simple manipulations, that the rate of change of the entropy is given by

$$
\dot{S}=k_{B} \iint d v d v^{\prime} k\left(v^{\prime} \rightarrow v\right) P\left(v^{\prime}, t\right) \ln \frac{P\left(v^{\prime}, t\right)}{P(v, t)} .
$$

This rate of entropy change can thus be rewritten under the standard form $\dot{S}=\dot{S}_{i}+\dot{S}_{e}$ with the rates of "entropy production" and "entropy exchange" given by

$$
\begin{aligned}
& \dot{S}_{i}=k_{B} \iint d v d v^{\prime} k\left(v^{\prime} \rightarrow v\right) P\left(v^{\prime}, t\right) \ln \frac{k\left(v^{\prime} \rightarrow v\right) P\left(v^{\prime}, t\right)}{k\left(v \rightarrow v^{\prime}\right) P(v, t)} \geqslant 0, \\
& \dot{S}_{e}=k_{B} \iint d v d v^{\prime} k\left(v^{\prime} \rightarrow v\right) P\left(v^{\prime}, t\right) \ln \frac{k\left(v \rightarrow v^{\prime}\right)}{k\left(v^{\prime} \rightarrow v\right)} .
\end{aligned}
$$

These results are mathematically exact, but in order to achieve a correct thermodynamic interpretation of the entropy production and exchange, one needs in addition proper physical input about the collision mechanism, i.e., about the collision rate. We focus here on the simplest case in which the collision process represents energy exchange with a single isotropic thermal reservoir at temperature $T$. As a result the collision process must induce, in absence of an external force, a relaxation to the Maxwell-Boltzmann distribution $\varphi_{0}$; i.e., one has

$$
\int d v^{\prime} k\left(v \rightarrow v^{\prime}\right) \varphi_{0}(v)=\int d v^{\prime} k\left(v^{\prime} \rightarrow v\right) \varphi_{0}\left(v^{\prime}\right),
$$

with

$$
\varphi_{0}(v)=\frac{e^{-v^{2} / 2 \sigma^{2}}}{\sigma \sqrt{2 \pi}}, \quad \sigma^{2}=m k_{B} T
$$

As was realized first by Onsager [21], microreversibility leads to a more stringent condition of detailed balance:

$$
k\left(v \rightarrow v^{\prime}\right) \varphi_{0}(v)=k\left(-v^{\prime} \rightarrow-v\right) \varphi_{0}\left(-v^{\prime}\right) .
$$

This detailed balance relation involves velocity inversion and seems to be at variance with the condition Eq. (9). The discrepancy is solved by making the crucial observation that, for a collision describing heat exchange with an isotropic stationary bath, there is an additional symmetry requirement of invariance under reflection (and more generally under rotation $[22,23])$ :

$$
k\left(v^{\prime} \rightarrow v\right)=k\left(-v^{\prime} \rightarrow-v\right) .
$$

With this extra condition, the detailed balance relation Eq. (11) implies Eq. (9). We stress that this extra condition reflects a property of the thermal bath. It is supposed to be isotropic, quasistatic, and in rest with respect to the system. Otherwise, other irreversible processes can take place and need to be identified. For example, the case of a bath in relative motion with respect to the system gives rise to momentum transfer with a corresponding irreversible entropy producing contribution; see, for example, Ref. [24]. Equation (11) allows us to make the consistent connection between first and second laws: the entropy exchange $\dot{S}_{e}$ can be rewritten $\left[\varphi_{0}(-v)=\varphi_{0}(v)\right]$ :

$$
\dot{S}_{e}=k_{B} \iint d v d v^{\prime} k\left(v^{\prime} \rightarrow v\right) P\left(v^{\prime}, t\right) \ln \frac{\varphi_{0}\left(v^{\prime}\right)}{\varphi_{0}(v)}=\frac{\dot{Q}}{T},
$$

where $\dot{Q}$ is the rate of energy (heat) exchange from the bath to the particle, cf. Eqs. (5) and (9). The entropy production is zero if and only if $k\left(v^{\prime} \rightarrow v\right) P\left(v^{\prime}\right)=k\left(v \rightarrow v^{\prime}\right) P(v)$, implying that $P(v) / P\left(v^{\prime}\right)=\varphi_{0}(v) / \varphi_{0}\left(v^{\prime}\right)$ and hence $P(v)=\varphi_{0}(v)$. We conclude that entropy production vanishes if and only if detailed balance is satisfied.

We now show that both Eqs. (11) and (12) are crucial to formulate the second law at the trajectory level. The stochastic entropy for the velocity variables reads [9]

$$
s(t)=-k_{B} \ln P[v(t), t] .
$$

Note that this entropy still retains an ensemble character, as one needs to specify the probability distribution $P(v, t)$, which is the probability to observe the particle with velocity $v$ at time $t$ starting from some specific initial probability distribution. This so-called forward experiment is run from initial time $t_{0}$ to some final time $t_{f}$. We now write

$$
\dot{s}=\dot{s}_{i}+\dot{s}_{e},
$$

where the trajectory entropy exchange is the obvious analog of the ensemble value given in Eq. (13): $\dot{s}_{e}=\dot{q} / T$. The meaning of the trajectory entropy production is most easily clarified by integrating Eq. (15) over a finite time, leading to the finite difference balance:

$$
\Delta s=\Delta_{i} s+\Delta_{e} s,
$$

with $\Delta_{e} s=q / T$ and $q$ is the total amount of heat received (by collisions) from the heat bath in the realization under consideration. An elegant derivation of the celebrated fluctuation theorem for the trajectory entropy production proceeds with the consideration of the probability for a trajectory in forward and reverse dynamics. We consider the simplest case 

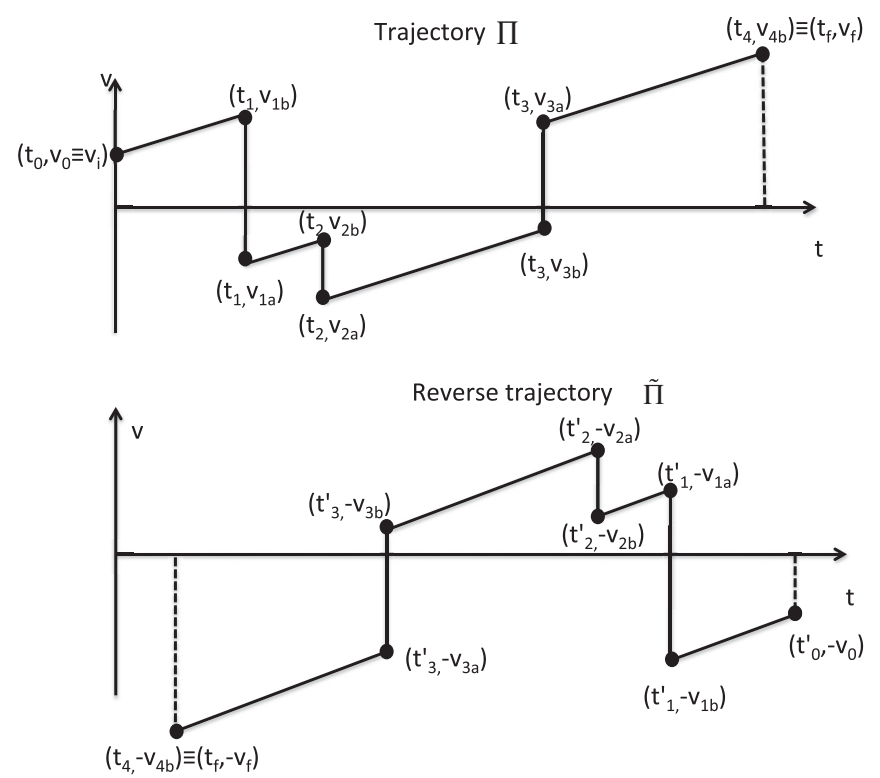

FIG. 1. Forward and reverse trajectories. In the forward trajectory $\Pi$ we start at time $t_{0}$ with a velocity $v_{0}$ and advance in time with acceleration $a$ until a collision with the bath occurs at time $t_{1}$ where the velocity before the collision is $v_{1 b}=v_{0}+a\left(t_{1}-t_{0}\right)$; then at time $t_{1}$ there is a collision that brings the velocity to $v_{1 a}$; the process repeats until a selected final time $t_{f}$ with a final velocity $v_{4 b}=v_{3 a}+$ $a\left(t_{4}-t_{3}\right) \equiv v_{f}$. In this particular example trajectory there were three collisions at times $t_{1}, t_{2}, t_{3}$. For the reverse trajectory $\tilde{\Pi}$ we start at time $t_{f}$ with the reverse velocity $-v_{f}$ and then advance in time with the same acceleration $a$; at time $t_{3}^{\prime}$, which is such that $t_{3}^{\prime}-t_{4}=t_{4}-$ $t_{3}$, the velocity is $v_{3}^{\prime}=-v_{4 b}+a\left(t_{3}^{\prime}-t_{4}\right)=-v_{3 a}$; then the collision with the bath selects precisely speed $-v_{3 b}$ and the velocity increases with acceleration $a$ until the time of the next collision $t_{2}^{\prime}-t_{3}^{\prime}=t_{3}-$ $t_{2}$, etc., arriving at a time $t_{0}^{\prime}=t_{f}+\left(t_{f}-t_{0}\right)$ at the reverse original speed, $-v_{0}$.

of steady-state operation, with the initial state of the forward experiment under acceleration $a$ sampled from the steady state distribution $P_{\mathrm{st}}(v, a)$. The reverse trajectory proceeds under the same acceleration $a$, starting with the final distribution of the forward probability, but with inverted speeds. Its properties will be identified with a superscript tilde. Let $P(\Pi)$ and $\tilde{P}(\tilde{\Pi})$ denote the probabilities for a forward and reverse trajectory, $\Pi$ and $\tilde{\Pi}$, respectively; see Fig. 1 . One now verifies the following striking equality:

$$
\Delta_{i} s=k_{B} \ln \frac{P(\Pi)}{\tilde{P}(\tilde{\Pi})} .
$$

The proof goes as follows. The probability of a trajectory involves the initial probability, the probability for not having collisions in-between the transitions, and the probability for transitions. Since the starting probability of the reverse dynamics is equal to the final probability of the direct dynamics, the logarithm of the ratio of the initial probability contributions reproduces $\Delta s=-k_{B} \ln P\left(v_{f}, t_{f}\right)+k_{B} \ln P\left(v_{0}, t_{0}\right)$; cf. Eq. (14). Due to the detailed balance condition Eq. (11), the $\log$ ratio of probabilities for collisions in forward and backward dynamics, cf. $\ln \frac{k\left(v^{\prime} \rightarrow v\right)}{k\left(-v \rightarrow-v^{\prime}\right)}=\ln \varphi_{0}(v) / \varphi_{0}\left(v^{\prime}\right)=$ $m\left(v^{\prime 2}-v^{2}\right) /\left(2 k_{B} T\right)$, reproduces $-\Delta_{e} s=-q / T$. Finally, due to the reflection symmetry Eq. (12), the probability for having no collisions, determined by the rates $k\left(v^{\prime} \rightarrow v\right)$ and $k\left(-v^{\prime} \rightarrow-v\right)$ when we have a velocity $v^{\prime}$ and $-v^{\prime}$, respectively, is the same in forward and backward trajectories. Without the parity property, this is no longer the case, and as mentioned in the introduction, the theory appears to become much more involved $[12,13]$. Hence the corresponding terms cancel out, and we have $\Delta_{i} s=\Delta s-\Delta_{e} s$ as required. We conclude that both at the ensemble level and at the trajectory level, the combination of detailed balance condition with the reflection symmetry are essential for a consistent stochastic thermodynamic interpretation.

The implications of Eq. (17) are well known [25]: the probability distributions $P\left(\Delta_{i} s\right)$ and $\tilde{P}\left(-\Delta_{i} s\right)$ for observing an entropy production $\Delta_{i} s$ in the forward process and minus this value in the backward process obey a detailed fluctuation theorem:

$$
\frac{P\left(\Delta_{i} s\right)}{\tilde{P}\left(-\Delta_{i} s\right)}=\exp \left(\Delta_{i} s / k_{B}\right),
$$

from which follows the integral fluctuation theorem: $\left\langle\exp \left(-\Delta_{i} s / k_{B}\right)\right\rangle=1$. A comment concerning the interpretation of Eq. (18) is in place; for more details see Refs. [10,11,2630]. In general, $-\Delta_{i} s$ is not the entropy production of the reverse trajectory. This will only be the case if the inverse "tilde" process is an involution, i.e., twice this operation is equal to the identity. In particular, the final probability distribution of the reverse process should be equal to the initial distribution of the forward process. In the case of even variables, a sufficient condition is that the forward process starts and ends in a steady state. For odd variables, this condition is not sufficient as is illustrated by the above example: the velocity inversion at the end of the forward process produces a probability distribution that is no longer at the steady state when $a \neq 0$. There is, however, a simple albeit somewhat artificial procedure to cure this problem and to obtain a detailed fluctuation theorem which is, just like the integral fluctuation theorem, expressed solely in terms of a (slightly modified) forward process. At the end of the forward process, one performs an instantaneous switch of the probability distribution from $P\left(v_{f}\right)$ to $P\left(-v_{f}\right)$, implying and entropy change of $\Delta_{v i} s=\ln P\left(v_{f}\right) / P\left(-v_{f}\right)$. This is, on average [with respect to $P\left(v_{f}\right)$ ], an irreversible entropy producing step. With this additional step, velocity inversion at the end of the forward will reproduce the steady state distribution, which is also in the case considered here the initial distribution of the forward process. In conclusion the corrected entropy production $\Delta_{i} s_{c}=\Delta_{i} s+\Delta_{v i} s$ will obey a symmetric detailed fluctuation theorem:

$$
\frac{P\left(\Delta_{i} s_{c}\right)}{P\left(-\Delta_{i} s_{c}\right)}=\exp \left(\Delta_{i} s_{c} / k_{B}\right)
$$

which has the advantage that it can conveniently be verified by considering statistics of the forward experiment alone. In Appendix A we present an independent proof of this fluctuation theorem without the explicit use of direct and reverse paths. 


\section{KANGAROO PROCESSES}

To illustrate the above formalism, we focus on the simple case of a "kangaroo" kinetic equation with a rate $k\left(v^{\prime} \rightarrow\right.$ v) [15]:

$$
k\left(v^{\prime} \rightarrow v\right)=\lambda\left(v^{\prime}\right) \varphi(v) .
$$

One verifies that the detailed balance symmetry Eq. (11) implies in this case that the collision rate $\lambda=1 / \tau$ is a constant, independent of $v^{\prime}$, and hence $k\left(v^{\prime} \rightarrow v\right)=\varphi_{0}(v) / \tau$. The reflection symmetry Eq. (12) is, in this case, an automatic consequence of the detailed balance condition Eq. (11). Numerical simulations of the stochastic process Eq. (1) (see Appendix $\mathrm{B}$ for additional details about the simulations) allow us to compute the probability distribution $P\left(\Delta_{i} s_{c}\right)$. In Fig. 2 we plot the probability distribution function $P\left(\Delta_{i} s_{c}\right)$ at different final times $t_{f}$. The validity of the fluctuation theorem is shown in Fig. 4, which collects data from all times. In the inset of Fig. 2 we plot the large deviation function $\Phi_{t}(x)$ that results directly from the fit $P\left(\Delta_{i} s_{c}\right) \sim \exp \left[-t \Phi_{t}\left(\Delta_{i} s_{c} / t\right)\right]$, with $t=t_{f}-t_{0}$, and test that the function $\Phi_{t}(x)$ tends to the asymptotic limit, the large deviation function, independent of time $t$. Note that the lack of convexity of the large deviation function for large values of the abscissa seems to disappear for increasing time and, hence, appears to be a finite-time effect.

To stress the need for a correct physical interpretation of the different expressions, we have also considered the case $\lambda(v)=\alpha|v|$ but still $\varphi(v)=\varphi_{0}(v)$, the Boltzmann-Maxwell distribution Eq. (11) for the velocities acquired from the bath. As these rates satisfy the symmetry condition Eq. (12) it is

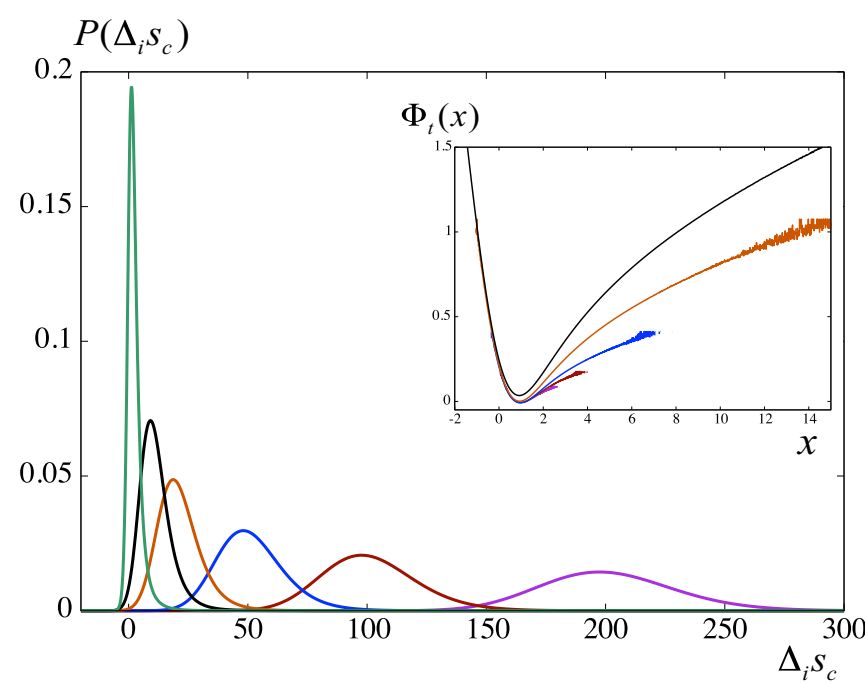

FIG. 2. (Color online) Probability distribution $P\left(\Delta_{i} s_{c}\right)$ (main plot) and large deviation function $\Phi_{t}(x)$ (inset) obtained from a numerical simulation of the stochastic process Eq. (1) for the case of a kangaroo reaction rate Eq. (20) with a uniform rate $\lambda(v)=1 / \tau$, and operating under steady-state conditions. We have taken $\tau=1$, the acceleration $a=1$ and $\sigma=1$ in Eq. (9) as well as the Boltzmann's constant $k_{B}=1$. From left to right in the main plot the curves correspond to $t_{f}=1,10,20,50,100,200$. In the inset we see that $\Phi_{t}(x)$ converges for large time to a time-independent curve, the large deviation function. The histograms have been obtained after averaging for $4 \times 10^{11}$ realizations.

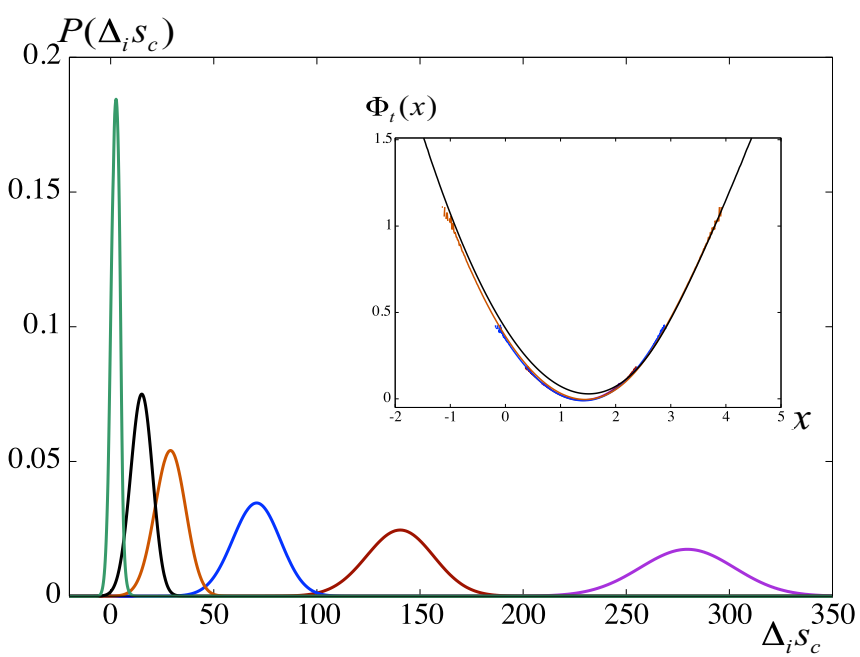

FIG. 3. (Color online) Similar to Fig. 2 for the case of a rate proportional to the absolute value of the velocity $\lambda(v)=\alpha|v|$ with $\alpha=1$. Same parameter values and time sequence as in Fig. 2 .

possible to formally derive the fluctuation theorem Eq. (19). The corresponding results for $P\left(\Delta_{i} s_{c}\right)$ and the large deviation function are shown in Fig. 3 whose data have been included in the analysis of Fig. 4, serving as a numerical test of the validity of the fluctuation theorem. However, the detailed balance condition is violated. The steady-state solution is not Maxwellian, and the interpretation of $\Delta_{i} s_{c}$ as thermodynamic entropy production is false.

\section{CONCLUSIONS}

We close with a few remarks. In hindsight, it is not surprising that a "parity" condition is required for a consistent stochastic thermodynamic interpretation. Indeed, every source of irreversibility has to be properly identified. The parity symmetry could be violated by considering, for example, a reservoir that is in motion with respect to the system.

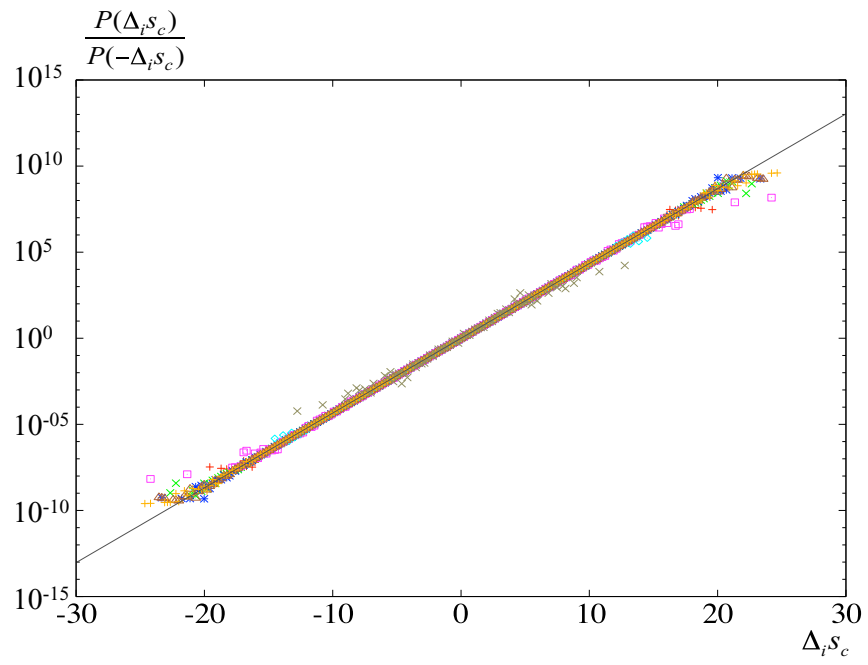

FIG. 4. (Color online) Test of the fluctuation theorem, including the data from the histograms of Figs. 2 and 3. 
In such a case, there will however be momentum transfer with a corresponding irreversible entropy production [24]. Stochastic thermodynamics has been developed in great detail for Langevin equations, see, e.g., Refs. [9,31,32], both in the over-damped and underdamped. A well-documented case is a chain of harmonic oscillators in contact with two heat baths; see Refs. [33-36]. One may wonder why the symmetry property Eq. (11) has not been discussed in this context. By making the diffusion approximation on the master Eq. (1) [14], one easily verifies that Eq. (11) requires that the drift term be uneven in the velocity and the noise term even. These conditions are met in a generic Langevin equation, explaining why this issue has not appeared in this context. The formalism presented above can be easily extended to more complicated situations, such as multiple particles with vectorial velocities in contact with several reservoirs of heat, particles, or momentum and with time-dependent external forcing. Also, the splitting of the entropy production in several components, such as the adiabatic and nonadiabatic contribution, proceeds as before [25].

\section{ACKNOWLEDGMENTS}

We acknowledge financial support from Ministerio de Economía y Competitividad (Spain) and Fondo Europeo de Desarrollo Regional under Project No. FIS2012-30634 (INTENSE@COSYP) and European Social Fund and Govern de les Illes Balears under programs Grups Competitius.

\section{APPENDIX A: DIRECT PROOF OF $\frac{P\left(\Delta_{i} s_{c}\right)}{P\left(-\Delta_{i} s_{c}\right)}=e^{\Delta_{i} s_{c}}$}

We present now a direct proof of the fluctuation theorem for the corrected entropy production $\Delta_{i} s_{c}$, which does not involve the consideration of a reverse trajectory. The only assumption we make in the derivation is that the rates satisfy the additional symmetry condition as given by Eq. (12). For the sake of clarity we consider separately the case in which there are no collisions in the trajectory and the case in which there is one collision. The case of an arbitrary number of collisions follows straightforwardly.

\section{A. Case of no collisions}

In the case of no collisions in the $(0, t)$ time interval, the final velocity is $v_{f}=v+a t$, with $v=v_{0}$ the initial velocity. The corrected entropy production is $\Delta_{i} s_{c}=\ln \frac{P_{\mathrm{st}}\left(v_{0}\right)}{P_{\mathrm{st}}\left(-v_{f}\right)}$ (we set $k_{B}=1$ in this appendix). To stress the dependence on the value of the acceleration $a$ we write $P_{\mathrm{st}}(v, a)$ for the stationary distribution and $P_{a}\left(\Delta_{i} s_{c}\right)$ for the probability distribution of the corrected entropy production. Using $P_{\mathrm{st}}(-v, a)=P_{\mathrm{st}}(v,-a)$, the latter can be computed as

$$
P_{a}\left(\Delta_{i} s_{c}\right)=\left\langle\delta\left(\Delta_{i} s_{c}-\ln \frac{P_{\mathrm{st}}(v, a)}{P_{\mathrm{st}}(v+a t,-a)}\right)\right\rangle_{v},
$$

with the average understood over the (steady-state) distribution of initial velocities:

$$
P_{a}\left(\Delta_{i} s_{c}\right)=\int d v P_{\mathrm{st}}(v, a) \delta\left[\Delta_{i} s_{c}-\ln \frac{P_{\mathrm{st}}(v, a)}{P_{\mathrm{st}}(v+a t,-a)}\right] .
$$

First we prove that $P_{-a}\left(\Delta_{i} s_{c}\right)=P_{a}\left(\Delta_{i} s_{c}\right)$, which makes complete physical sense. Using again $P_{\mathrm{st}}(v, a)=P_{\mathrm{st}}(-v,-a)$,

$$
\begin{aligned}
P_{-a}\left(\Delta_{i} s_{c}\right) & =\int d v P_{\mathrm{st}}(v,-a) \delta\left[\Delta_{i} s_{c}-\ln \frac{P_{\mathrm{st}}(v,-a)}{P_{\mathrm{st}}(v-a t, a)}\right] \\
& =\int d v P_{\mathrm{st}}(-v, a) \delta\left[\Delta_{i} s_{c}-\ln \frac{P_{\mathrm{st}}(-v, a)}{P_{\mathrm{st}}(-v+a t,-a)}\right] \\
& =\int d v P_{\mathrm{st}}(v, a) \delta\left[\Delta_{i} s_{c}-\ln \frac{P_{\mathrm{st}}(v, a)}{P_{\mathrm{st}}(v+a t,-a)}\right] \\
& =-v \int P_{a}\left(\Delta_{i} s_{c}\right) .
\end{aligned}
$$

Now in Eq. (A2) we insert $e^{\Delta_{i} s_{c}} e^{-\Delta_{i} s_{c}}$ and use the $\delta$ function in the second term:

$$
\begin{aligned}
P_{a}\left(\Delta_{i} s_{c}\right) & =\exp \left(\Delta_{i} s_{c}\right) \int d v P_{\mathrm{st}}(v, a) \exp \left(-\Delta_{i} s_{c}\right) \delta\left[\Delta_{i} s_{c}-\ln \frac{P_{\mathrm{st}}(v, a)}{P_{\mathrm{st}}(v+a t,-a)}\right] \\
& =\exp \left(\Delta_{i} s_{c}\right) \int d v P_{\mathrm{st}}(v, a) \frac{P_{\mathrm{st}}(v+a t,-a)}{P_{\mathrm{st}}(v, a)} \delta\left[\Delta_{i} s_{c}-\ln \frac{P_{\mathrm{st}}(v, a)}{P_{\mathrm{st}}(v+a t,-a)}\right] \\
& =\exp \left(\Delta_{i} s_{c}\right) \int d v P_{\mathrm{st}}(v,-a) \delta\left[\Delta_{i} s_{c}-\ln \frac{P_{\mathrm{st}}(v-a t, a)}{P_{\mathrm{st}}(v,-a)}\right] \\
& =\operatorname{at} \rightarrow v \\
\delta(x)=\delta(-x) & \exp \left(\Delta_{i} s_{c}\right) \int d v P_{\mathrm{st}}(v,-a) \delta\left[-\Delta_{i} s_{c}-\ln \frac{P_{\mathrm{st}}(v,-a)}{P_{\mathrm{st}}(v-a t, a)}\right] \\
& =\exp \left(\Delta_{i} s_{c}\right) P_{-a}\left(-\Delta_{i} s_{c}\right) \\
& =\exp \left(\Delta_{i} s_{c}\right) P_{a}\left(-\Delta_{i} s_{c}\right),
\end{aligned}
$$


the desired fluctuation theorem.

\section{B. Case of one collision}

We again call $v$ the initial velocity at $t=0$. Suppose that a collision takes place at time $t_{1}$, when the velocity is $v+a t_{1}$. A new velocity $v_{1}$ is drawn from the distribution $k\left(v+a t_{1} \rightarrow v_{1}\right)$. The particle undergoes again the constant acceleration $a$, resulting in a final velocity $v_{1}+a\left(t_{f}-t_{1}\right)$ at the final time $t_{f}$. The corrected entropy production including the collision term is [see also Eq. (B2)], later,

$$
\Delta_{i} s_{c}=\ln \left[\frac{P_{\mathrm{st}}(v, a)}{P_{\mathrm{st}}\left(v_{1}+a\left(t_{f}-t_{1}\right),-a\right)}\right]+\ln \left[\frac{k\left(v+a t_{1} \rightarrow v_{1}\right)}{k\left(v_{1} \rightarrow v+a t_{1}\right)}\right] .
$$

Its probability density reads

$$
\begin{aligned}
P_{a}\left(\Delta_{i} s_{c}\right)= & \int d v P_{\mathrm{st}}(v, a) \int d v_{1} \int_{0}^{t_{f}} d t_{1} k\left(v+a t_{1} \rightarrow v_{1}\right) \\
& \times \delta\left\{\Delta_{i} s_{c}-\ln \left[\frac{P_{\mathrm{st}}(v, a)}{P_{\mathrm{st}}\left(v_{1}+a\left(t_{f}-t_{1}\right),-a\right)} \frac{k\left(v+a t_{1} \rightarrow v_{1}\right)}{k\left(v_{1} \rightarrow v+a t_{1}\right)}\right]\right\} .
\end{aligned}
$$

We first prove that $P_{a}\left(\Delta_{i} s_{c}\right)=P_{-a}\left(\Delta_{i} s_{c}\right)$. We use $P_{\mathrm{st}}(v, a)=P_{\mathrm{st}}(-v,-a)$ and Eq. (12):

$$
\begin{aligned}
P_{-a}\left(\Delta_{i} s_{c}\right)= & \iiint d v d v_{1} d t_{1} P_{\mathrm{st}}(v,-a) k\left(v-a t_{1} \rightarrow v_{1}\right) \\
& \times \delta\left\{\Delta_{i} s_{c}-\ln \left[\frac{P_{\mathrm{st}}(v,-a)}{P_{\mathrm{st}}\left(v_{1}-a\left(t_{f}-t_{1}\right), a\right)} \frac{k\left(v-a t_{1} \rightarrow v_{1}\right)}{k\left(v_{1} \rightarrow v-a t_{1}\right)}\right]\right\} \\
= & \iiint d v d v_{1} d t_{1} P_{\mathrm{st}}(-v, a) k\left(-v+a t_{1} \rightarrow-v_{1}\right) \\
& \times \delta\left\{\Delta_{i} s_{c}-\ln \left[\frac{P_{\mathrm{st}}(-v, a)}{P_{\mathrm{st}}\left(-v_{1}+a\left(t_{f}-t_{1}\right),-a\right)} \frac{k\left(-v+a t_{1} \rightarrow-v_{1}\right)}{k\left(-v_{1} \rightarrow-v+a t_{1}\right)}\right]\right\} \\
& =-v \\
v_{1} \rightarrow-v_{1} & \iiint d v d v_{1} d t_{1} P_{\mathrm{st}}(v, a) k\left(v+a t_{1} \rightarrow v_{1}\right) \\
& \times \delta\left\{\Delta_{i} s_{c}-\ln \left[\frac{P_{\mathrm{st}}(v, a)}{P_{\mathrm{st}}\left(v_{1}+a\left(t_{f}-t_{1}\right),-a\right)} \frac{k\left(v+a t_{1} \rightarrow v_{1}\right)}{k\left(v_{1} \rightarrow v+a t_{1}\right)}\right]\right\} \\
= & P_{a}\left(\Delta_{i} s_{c}\right) .
\end{aligned}
$$

For the proof of the fluctuation theorem we insert $e^{\Delta_{i} s_{c}} e^{-\Delta_{i} s_{c}}$ and replace inside the integral by using the $\delta$ function:

$$
\begin{aligned}
P_{a}\left(\Delta_{i} s_{c}\right)= & e^{\Delta_{i} s_{c}} \iiint d v d v_{1} d t_{1} P_{\mathrm{st}}(v, a) k\left(v+a t_{1} \rightarrow v_{1}\right) e^{-\Delta_{i} s_{c}} \\
& \times \delta\left\{\Delta_{i} s_{c}-\ln \left[\frac{P_{\mathrm{st}}(v, a)}{P_{\mathrm{st}}\left(v_{1}+a\left(t_{f}-t_{1}\right),-a\right)} \frac{k\left(v+a t_{1} \rightarrow v_{1}\right)}{k\left(v_{1} \rightarrow v+a t_{1}\right)}\right]\right\} \\
= & e^{\Delta_{i} s_{c}} \iiint d v d v_{1} d t_{1} P_{\mathrm{st}}(v, a) k\left(v+a t_{1} \rightarrow v_{1}\right) \frac{P_{\mathrm{st}}\left(v_{1}+a\left(t_{f}-t_{1}\right),-a\right)}{P_{\mathrm{st}}(v, a)} \frac{k\left(v_{1} \rightarrow v+a t_{1}\right)}{k\left(v+a t_{1} \rightarrow v_{1}\right)} \\
& \times \delta\left\{\Delta_{i} s_{c}-\ln \left[\frac{P_{\mathrm{st}}(v, a)}{P_{\mathrm{st}}\left(v_{1}+a\left(t_{f}-t_{1}\right),-a\right)} \frac{k\left(v+a t_{1} \rightarrow v_{1}\right)}{k\left(v_{1} \rightarrow v+a t_{1}\right)}\right]\right\} \\
= & e^{\Delta_{i} s_{c}} \iiint d v d v_{1} d t_{1} P_{\mathrm{st}}\left(v_{1}+a\left(t_{f}-t_{1}\right),-a\right) k\left(v_{1} \rightarrow v+a t_{1}\right) \\
& \times \delta\left\{\Delta_{i} s_{c}-\ln \left[\frac{P_{\mathrm{st}}(v, a)}{P_{\mathrm{st}}\left(v_{1}+a\left(t_{f}-t_{1}\right),-a\right)} \frac{k\left(v+a t_{1} \rightarrow v_{1}\right)}{k\left(v_{1} \rightarrow v+a t_{1}\right)}\right]\right\} .
\end{aligned}
$$

Now we change $v^{\prime} \equiv v_{1}+a\left(t_{f}-t_{1}\right)$ and $v_{1}^{\prime} \equiv v+a t_{1}$ (and drop the primes in the resulting expression),

$$
\begin{aligned}
P_{a}\left(\Delta_{i} s_{c}\right)= & e^{\Delta_{i} s_{c}} \iiint d v d v_{1} d t_{1} P_{\mathrm{st}}(v,-a) k\left[v-a\left(t_{f}-t_{1}\right) \rightarrow v_{1}\right] \\
& \times \delta\left(\Delta_{i} s_{c}-\ln \left\{\frac{P_{\mathrm{st}}\left(v_{1}-a t_{1}, a\right)}{P_{\mathrm{st}}(v,-a)} \frac{k\left[v-a\left(t_{f}-t_{1}\right) \rightarrow v_{1}\right]}{k\left[v_{1} \rightarrow v-a\left(t_{f}-t_{1}\right)\right]}\right\}\right) .
\end{aligned}
$$


We replace $t_{1}^{\prime} \equiv t_{f}-t_{1}$ and use $\int_{0}^{t_{f}} d t_{1}=\int_{0}^{t_{f}} d t_{1}^{\prime}$ (and again drop the prime) to obtain

$$
\begin{aligned}
P_{a}\left(\Delta_{i} s_{c}\right)= & e^{\Delta_{i} s_{c}} \iiint d v d v_{1} d t_{1} P_{\mathrm{st}}(v,-a) k\left(v-a t_{1} \rightarrow v_{1}\right) \\
& \times \delta\left(\Delta_{i} s_{c}-\ln \left\{\frac{P_{\mathrm{st}}\left[v_{1}-a\left(t_{f}-t_{1}\right), a\right]}{P_{\mathrm{st}}(v,-a)} \frac{k\left(v-a t_{1} \rightarrow v_{1}\right)}{k\left(v_{1} \rightarrow v-a t_{1}\right)}\right\}\right) \\
\begin{array}{c}
\delta(x)=\delta(-x) \\
=
\end{array} & e^{\Delta_{i} s_{c}} \iiint d v d v_{1} d t_{1} P_{\mathrm{st}}(v,-a) k\left(v-a t_{1} \rightarrow v_{1}\right) \\
& \times \delta\left(-\Delta_{i} s_{c}-\ln \left\{\frac{P_{\mathrm{st}}(v,-a)}{P_{\mathrm{st}}\left[v_{1}-a\left(t_{f}-t_{1}\right), a\right]} \frac{k\left(v_{1} \rightarrow v-a t_{1}\right)}{k\left(v-a t_{1} \rightarrow v_{1}\right)}\right\}\right) \\
= & e^{\Delta_{i} s_{c}} P_{-a}\left(-\Delta_{i} s_{c}\right) \\
= & e^{\Delta_{i} s_{c}} P_{a}\left(-\Delta_{i} s_{c}\right),
\end{aligned}
$$

again the fluctuation theorem.

\section{APPENDIX B: DETAILS OF NUMERICAL SIMULATIONS}

The entropy production $S_{i}\left(t_{f}\right)-S_{i}\left(t_{0}\right)$ can be considered as the ensemble average of the entropy trajectory production $\Delta_{i} s$. A single trajectory $\Pi$ consists of an initial velocity $v_{0}$ taken from the stationary distribution $P_{\mathrm{st}}(v)$, a series of collisions at times $t_{1}, t_{2}, \ldots$, and a final value $v_{f}$ at time $t_{f}$; see Fig. 1 for details.

The trajectory entropy is defined as

$$
\Delta_{i} s=\ln \frac{P_{\mathrm{st}}\left(v_{0}\right)}{P_{\mathrm{st}}\left(v_{f}\right)}+\sum_{\text {collisiontimes }_{i}, i=1,2, \ldots} \ln \frac{k\left(v_{i b} \rightarrow v_{i a}\right)}{k\left(v_{i a} \rightarrow v_{i b}\right)}
$$

where $v_{i b}$ stands for $v_{i}^{\text {beforecollision }}$ and $v_{i a}$ for $v_{i}^{\text {aftercollision }}$. As we have split $k\left(v \rightarrow v^{\prime}\right)=\lambda(v) \varphi\left(v^{\prime}\right)$ we can write

$$
\Delta_{i} s=\ln \frac{P_{\mathrm{st}}\left(v_{0}\right)}{P_{\mathrm{st}}\left(v_{f}\right)}+\sum_{\text {collisiontimest } \mathrm{i}, \mathrm{i}=1,2, \ldots} \ln \frac{\lambda\left(v_{i b}\right) \varphi\left(v_{i a}\right)}{\lambda\left(v_{i a}\right) \varphi\left(v_{i b}\right)} .
$$

The corrected entropy production $\Delta_{i} s_{c}=\Delta_{i} s+\Delta_{v i} s$ is

$$
\Delta_{i} s_{c}=\ln \frac{P_{\mathrm{st}}\left(v_{0}\right)}{P_{\mathrm{st}}\left(-v_{f}\right)}+\sum_{\text {collisiontimest } \mathrm{i}, \mathrm{i}=1,2, \ldots} \ln \frac{\lambda\left(v_{i b}\right) \varphi\left(v_{i a}\right)}{\lambda\left(v_{i a}\right) \varphi\left(v_{i b}\right)} .
$$

A numerical simulation proceeds as follows:

Start with a value $v_{0}$ at $t_{0}=0$, obtained from $P_{\mathrm{st}}(v)$. For this we use the technique of inverting the cumulative distribution function, either analytically or numerically; see Ref. [37]. The stationary distribution is calculated for both the cases of constant rate $\lambda=1 / \tau$ or a rate proportional to the speed $\lambda=\alpha|v|$ as detailed in Appendix C. Then, compute $t_{1}$ solving the equation

$$
1-e^{-\int_{0}^{t_{1}} \lambda\left(v_{0}+a t\right) d t}=u_{1},
$$

or equivalently

$$
\int_{0}^{t_{1}} \lambda\left(v_{0}+a t\right) d t=-\ln \left(u_{1}\right)
$$

with $u_{i}$ independent random numbers uniformly distributed in $(0,1)$. Compute $v_{1 b}=v_{0}+a t_{1}$. Generate a new value $v_{1 a}$ sampled from $\varphi_{0}(v)$, i.e., a Gaussian distribution of zero mean and variance $\sigma^{2}$. Compute $t_{2}=t_{1}+\Delta t_{2}$ solving

$$
\int_{0}^{\Delta t_{2}} \lambda\left(v_{0}+a t\right) d t=-\ln \left(u_{2}\right) .
$$

Set $v_{2 b}=v_{1 a}+a\left(t_{2}-t_{1}\right)$. Generate a new value $v_{2 a}$ sampled from $\varphi(v)$. Keep on going until a prefixed time $t_{f}$ is surpassed. Then, compute the change of entropy of that trajectory $\Delta_{i} s$ using Eq. (B1).

\section{APPENDIX C: STEADY-STATE DISTRIBUTION}

Integrating Eq. (1) over the positions $x$ and replacing the kangaroo rates Eq. (20), we obtain

$$
\left[\frac{\partial}{\partial t}+a \frac{\partial}{\partial v}\right] P(v, t)=\varphi(v) \int d v^{\prime} \lambda\left(v^{\prime}\right) P\left(v^{\prime}, t\right)-\lambda(v) P(v, t) \text {. }
$$

Note that if $\varphi(-v)=\varphi(v)$ and $\lambda(-v)=\lambda(v)$, then the above equation in invariant under the transformation $v \rightarrow-v, a \rightarrow$ $-a$, and its solution will inherit this symmetry. The stationary state solution of this equation is

$$
P_{\mathrm{st}}(v)= \begin{cases}C^{-1} \int_{-\infty}^{v} d v^{\prime} \varphi\left(v^{\prime}\right) e^{-\frac{1}{a} \int_{v^{\prime}}^{v} d v^{\prime \prime} \lambda\left(v^{\prime \prime}\right)}, & a>0, \\ C^{-1} \int_{v}^{\infty} d v^{\prime} \varphi\left(v^{\prime}\right) e^{-\frac{1}{a} \int_{v^{\prime}}^{v} d v^{\prime \prime} \lambda\left(v^{\prime \prime}\right)}, & a<0,\end{cases}
$$

where $C$ is a normalization constant. We focus below on the cases of a constant collision rate and a rate proportional to the speed.

\section{A. Constant collision rate}

For a constant collision rate $\lambda=1 / \tau$, the evolution Eq. (C1) reduces to

$$
\left[\frac{\partial}{\partial t}+a \frac{\partial}{\partial v}\right] P(v, t)=\frac{1}{\tau}[\varphi(v)-P(v, t)],
$$

and the full time-dependent solution for a given initial condition $P(v, t=0)=P_{0}(v)$ can be obtained. We mention the following two equivalent forms,

$$
P(v, t)=e^{-t / \tau} P_{0}(v-a t)+\frac{1}{\tau} \int_{0}^{t} d t^{\prime} \varphi\left[v-a\left(t-t^{\prime}\right)\right] e^{-\frac{t-t^{\prime}}{\tau}},
$$


or [upon changing variables $v^{\prime}=v-a\left(t-t^{\prime}\right)$ in the integral],

$$
P(v, t)=e^{-t / \tau} P_{0}(v-a t)+\frac{1}{a \tau} \int_{v-a t}^{v} d v^{\prime} \varphi\left(v^{\prime}\right) e^{-\frac{v-v^{\prime}}{a \tau}} .
$$

The first moment of the velocity is found to be

$$
\langle v(t)\rangle=e^{-t / \tau}\langle v(0)\rangle+\left(1-e^{-t / \tau}\right)\left(a \tau+\langle v\rangle_{\varphi}\right),
$$

where $\langle.\rangle_{\varphi}$ refers to an average with respect to $\varphi$. One finds, as expected, that

$$
\lim _{t \rightarrow \infty}\langle v(t)\rangle=\langle v\rangle_{\varphi}+a \tau
$$

The general expression for the second moment is cumbersome, and we only mention the stationary-state value:

$$
\lim _{t \rightarrow \infty}\left\langle v(t)^{2}\right\rangle-\langle v(t)\rangle^{2}=\left\langle v^{2}\right\rangle_{\varphi}-\langle v\rangle_{\varphi}^{2}+a^{2} \tau^{2} .
$$

The stationary solution is obtained from Eq. (C2), or by taking in Eq. (C4) the limit $t \rightarrow \infty$ :

$$
P_{\mathrm{st}}(v)= \begin{cases}\frac{1}{a \tau} \int_{-\infty}^{v} d v^{\prime} \varphi\left(v^{\prime}\right) e^{-\frac{v-v^{\prime}}{a \tau}}, & a>0, \\ \frac{1}{-a \tau} \int_{v}^{\infty} d v^{\prime} \varphi\left(v^{\prime}\right) e^{-\frac{v-v^{\prime}}{a \tau}}, & a<0 .\end{cases}
$$

We finally mention the following explicit result for a Maxwell-Boltzmann distribution $\varphi=\varphi_{0}=\frac{1}{\sigma \sqrt{2 \pi}} e^{-\frac{v^{2}}{2 \sigma^{2}}}$ :

$$
\begin{aligned}
P_{\mathrm{st}}(v) & =\left\{\begin{array}{ll}
\frac{1}{2 a \tau} e^{\frac{\sigma^{2}-2 a v \tau}{2 a^{2} \tau^{2}}} \operatorname{erfc}\left(\frac{\sigma^{2}-\mathrm{av} \tau}{\sqrt{2} \mathrm{a} \sigma \tau}\right), & a>0 \\
\frac{1}{-2 a \tau} e^{\frac{\sigma^{2}-2 a v \tau}{2 a^{2} \tau^{2}}} \operatorname{erfc}\left(\frac{\sigma^{2}-\mathrm{av} \tau}{-\sqrt{2} \mathrm{a} \sigma \tau}\right), & a<0
\end{array}\right\} \\
& =\frac{1}{2|a| \tau} e^{\frac{\sigma^{2}-2 a v \tau}{2 a^{2} \tau^{2}}} \operatorname{erfc}\left(\frac{\sigma^{2}-\mathrm{av} \tau}{\sqrt{2}|\mathrm{a}| \sigma \tau}\right) .
\end{aligned}
$$

This expression displays the previously mentioned invariance under the transformation $(v ; a) \rightarrow(-v ;-a)$.

\section{B. Rate proportional to speed}

For the case of a rate proportional to the speed, $\lambda(v)=|v|$, the integral appearing in Eq. (C2) is quite complicated. We therefore focus immediately on the most relevant case of a Maxwellian distribution $\varphi_{0}(v)=\frac{1}{\sqrt{2 \pi}} e^{-\frac{v^{2}}{2}}$. A cumbersome calculation yields for $a>1$,

$$
P_{\mathrm{st}}(v, a)=C^{-1}(a) \times \begin{cases}e^{\frac{v^{2}}{2 a}}\left[1+\operatorname{erf}\left(\mathrm{v} \sqrt{\frac{\mathrm{a}+1}{2 \mathrm{a}}}\right)\right], & v \leqslant 0, \\ e^{-\frac{v^{2}}{2 a}}\left[1+\sqrt{\frac{a+1}{a-1}} \operatorname{erf}\left(\mathrm{v} \sqrt{\frac{\mathrm{a}-1}{2 \mathrm{a}}}\right)\right], & v \geqslant 0,\end{cases}
$$

while the result for $0<a<1$ is

$$
P_{\mathrm{st}}(v, a)=C^{-1}(a) \times \begin{cases}e^{\frac{v^{2}}{2 a}}\left[1+\operatorname{erf}\left(\mathrm{v} \sqrt{\frac{\mathrm{a}+1}{2 \mathrm{a}}}\right)\right], & v \leqslant 0, \\ e^{-\frac{v^{2}}{2 a}}\left[1+\sqrt{\frac{a+1}{1-a}} \operatorname{erfi}\left(\mathrm{v} \sqrt{\frac{1-\mathrm{a}}{2 \mathrm{a}}}\right)\right], & v \geqslant 0 .\end{cases}
$$

The limiting case $a=1$ is

$$
P_{\mathrm{st}}(v, 1)=C^{-1}(1) \times \begin{cases}e^{\frac{v^{2}}{2}}[1+\operatorname{erf}(\mathrm{v})], & v \leqslant 0, \\ e^{-\frac{v^{2}}{2}}\left(1+\frac{2}{\sqrt{\pi}} v\right), & v \geqslant 0 .\end{cases}
$$

The normalization constant is:

$$
C(a)= \begin{cases}\sqrt{\frac{a \pi}{2}}+\sqrt{\frac{2 a}{\pi}}\left[\operatorname{arctanh}\left(\frac{1}{\sqrt{1+\mathrm{a}}}\right)+\sqrt{\frac{1+\mathrm{a}}{\mathrm{a}-1}} \arctan (\sqrt{\mathrm{a}-1})\right], & a>1 \\ \sqrt{\frac{\pi}{2}}+\frac{2}{\sqrt{\pi}}+\sqrt{\frac{2}{\pi}} \operatorname{arctanh}\left(\frac{1}{\sqrt{2}}\right)=3.08493 \ldots, & a=1 . \\ \sqrt{\frac{a \pi}{2}}+\sqrt{\frac{2 a}{\pi}}\left[\operatorname{arctanh}\left(\frac{1}{\sqrt{1+\mathrm{a}}}\right)+\sqrt{\frac{1+\mathrm{a}}{1-\mathrm{a}}} \operatorname{arctanh}(\sqrt{1-\mathrm{a}})\right], & a<1\end{cases}
$$

For $a<0$ we can use the property $P_{\mathrm{st}}(v, a)=P_{\mathrm{st}}(-v,-a)$. A direct integration gives the first and second moments in the steady state. While the expression for the second moment is too long to be reproduced here, the first moment is related to the normalization constant as

$$
\langle v\rangle_{\mathrm{st}}=\frac{2 a}{C(a)} .
$$


[1] C. Jarzynski, Phys. Rev. Lett. 78, 2690 (1997).

[2] R. Kawai, J. M. R. Parrondo, and C. V. den Broeck, Phys. Rev. Lett. 98, 080602 (2007).

[3] D. J. Evans, E. G. D. Cohen, and G. P. Morriss, Phys. Rev. Lett. 71, 2401 (1993).

[4] G. Gallavotti, Phys. Rev. Lett. 77, 4334 (1996).

[5] G. Crooks, J. Stat. Phys. 90, 1481 (1998).

[6] J. Kurchan, J. Phys. A: Math. Gen. 31, 3719 (1998).

[7] J. Lebowitz and H. Spohn, J. Stat. Phys. 95, 333 (1999).

[8] S. Sekimoto, Stochastic Energetics (Springer, New York, 2010).

[9] U. Seifert, Rep. Progr. Phys. 75, 126001 (2012).

[10] C. Van den Broeck, in Proceedings of the International School of Physics "Enrico Fermi," Course CLXXXIV "Physics of Complex Colloids," edited by C. Bechinger, F. Sciortino, and Ziherl P. (Italian Physical Society, Bologna, 2013).

[11] C. Van den Broeck and M. Esposito, Physica A 418, 6 (2015).

[12] R. E. Spinney and I. J. Ford, Phys. Rev. Lett. 108, 170603 (2012).

[13] H. K. Lee, C. Kwon, and H. Park, Phys. Rev. Lett. 110, 050602 (2013).

[14] N. van Kampen, Stochastic Processes in Physics and Chemistry, 3rd ed. (North-Holland, Amsterdam, 2007).

[15] C. Van den Broeck and R. Toral, Phys. Rev. E 89, 062124 (2014).

[16] S. Deffner and A. Saxena, Phys. Rev. Lett. 114, 150601 (2015).

[17] G. Gradenigo, A. Puglisi, A. Sarracino, and U. M. B. Marconi, Phys. Rev. E 85, 031112 (2012).

[18] G. Gradenigo, A. Sarracino, A. Puglisi, and H. Touchette, J. Phys. A: Math. Theoret. 46, 335002 (2013).

[19] T. G. Sano and H. Hayakawa, Phys. Rev. E 89, 032104 (2014).

[20] M. Itami and S.-i. Sasa, J. Stat. Phys. 158, 37 (2015).
[21] R. Balescu, Equilibrium and Non-Equilibrium Statistical Mechanics (Wiley, New York, 1975).

[22] P. Gaspard, Physica A: Stat. Mech. Appl. 392, 639 (2013).

[23] P. B. Gaveau and M. Moreau, Eur. Phys. J. Special Topics 224, 905 (2015).

[24] K. Wood, C. Van den Broeck, R. Kawai, and K. Lindenberg, Phys. Rev. E 75, 061116 (2007).

[25] M. Esposito and C. Van den Broeck, Phys. Rev. Lett. 104, 090601 (2010).

[26] R. Spinney and I. Ford, in Nonequilibrium Statistical Physics of Small Systems: Fluctuation Relations and Beyond, edited by W. Klages and C. Jarzinsky (Wiley, New York, 2013).

[27] T. Becker, T. Willaert, B. Cleuren, and C. Van den Broeck, Phys. Rev. E 91, 012101 (2015).

[28] R. J. Harris and G. M. Schütz, J. Stat. Mech.: Theory Exp. (2007) P07020.

[29] R. García-García, V. Lecomte, A. B. Kolton, and D. Domínguez, J. Stat. Mech.: Theory Exp. (2012) P02009.

[30] Z. Gong and H. T. Quan, arXiv:1503.05036

[31] T. Tomé and M. J. de Oliveira, Phys. Rev. E 91, 042140 (2015).

[32] S. Lahiri and A. M. Jayannavar, Eur. Phys. J. B 87, 195 (2014).

[33] K. Saito and A. Dhar, Phys. Rev. Lett. 99, 180601 (2007).

[34] K. Saito and A. Dhar, Phys. Rev. E 83, 041121 (2011).

[35] A. Kundu, S. Sabhapandit, and A. Dhar, J. Stat. Mech.: Theory Exp. (2011) P03007.

[36] H. C. Fogedby and A. Imparato, J. Stat. Mech.: Theory Exp. (2012) P04005.

[37] R. Toral and P. Colet, Stochastic Numerical Methods: An Introduction for Students and Scientists (Wiley, New York, 2014). 